\begin{tabular}{|c|l|}
\hline Title & Semi-automated protein crystal mounting device for the sulfur single wavelength anomal ous diffraction method \\
\hline Author(s) & Kitago, Yu; Watanabe, Nobuhisa; Tanaka, Isao \\
\hline Citation & $\begin{array}{l}\text { Journal of A pplied Crystallography, 43(2), 341-346 } \\
\text { https://doi.org/40.1107/S0021889809054272 }\end{array}$ \\
\hline Issue Date & 2010-04 \\
\hline Doc URL & http://hdl.handle.net/2115/43006 \\
\hline Type & article \\
\hline File Information & watanabe_JAC43.pdf \\
\hline
\end{tabular}

Instructions for use 


\title{
Semi-automated protein crystal mounting device for the sulfur single-wavelength anomalous diffraction method
}

\author{
Yu Kitago, Nobuhisa Watanabe and Isao Tanaka
}

J. Appl. Cryst. (2010). 43, 341-346

Copyright (C) International Union of Crystallography

Author(s) of this paper may load this reprint on their own web site or institutional repository provided that this cover page is retained. Republication of this article or its storage in electronic databases other than as specified above is not permitted without prior permission in writing from the IUCr.

For further information see http://journals.iucr.org/services/authorrights.html

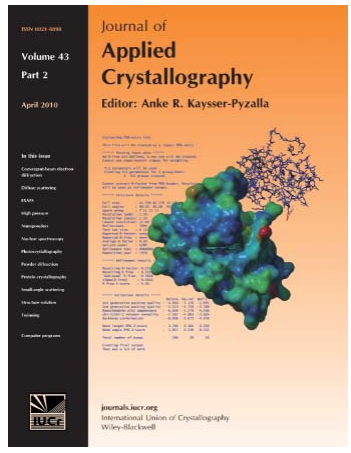

Many research topics in condensed matter research, materials science and the life sciences make use of crystallographic methods to study crystalline and non-crystalline matter with neutrons, X-rays and electrons. Articles published in the Journal of Applied Crystallography focus on these methods and their use in identifying structural and diffusioncontrolled phase transformations, structure-property relationships, structural changes of defects, interfaces and surfaces, etc. Developments of instrumentation and crystallographic apparatus, theory and interpretation, numerical analysis and other related subjects are also covered. The journal is the primary place where crystallographic computer program information is published.

\section{Crystallography Journals Online is available from journals.iucr.org}


Journal of

Applied

Crystallography

ISSN 0021-8898

Received 25 September 2009

Accepted 16 December 2009

C 2010 International Union of Crystallography Printed in Singapore - all rights reserved

\section{Semi-automated protein crystal mounting device for the sulfur single-wavelength anomalous diffraction method}

\author{
Yu Kitago, ${ }^{a}$ Nobuhisa Watanabe ${ }^{a, b, c *}$ and Isao Tanaka ${ }^{a}$ \\ ${ }^{\mathbf{a}}$ Faculty of Advanced Life Science, Hokkaido University, Sapporo 0600810, Japan, ${ }^{\mathbf{b}}$ Department of \\ Biotechnology, Graduate School of Engineering, Nagoya University, Nagoya 4648603, Japan, and \\ ${ }^{\mathbf{c}}$ Synchrotron Radiation Research Center, Nagoya University, Nagoya 4648603, Japan. Corre- \\ spondence e-mail: nobuhisa@nagoya-u.jp
}

\begin{abstract}
Use of longer-wavelength X-rays has advantages for the detection of small anomalous signals from light atoms, such as sulfur, in protein molecules. However, the accuracy of the measured diffraction data decreases at longer wavelengths because of the greater X-ray absorption. The capillary-top mounting method (formerly the loopless mounting method) makes it possible to eliminate frozen solution around the protein crystal and reduces systematic errors in the evaluation of small anomalous differences. However, use of this method requires custom-made tools and a large amount of skill. Here, the development of a device that can freeze the protein crystal semi-automatically using the capillary-top mounting method is described. This device can pick up the protein crystal from the crystallization drop using a micro-manipulator, and further procedures, such as withdrawal of the solution around the crystal by suction and subsequent flash freezing of the protein crystal, are carried out automatically. This device makes it easy for structural biologists to use the capillary-top mounting method for sulfur single-wavelength anomalous diffraction phasing using longer-wavelength X-rays.
\end{abstract}

\section{Introduction}

X-ray crystallography is an efficient method for determination of the three-dimensional structures of biological macromolecules, from which important information can be extracted. Over the past decade, protein crystallography techniques have become highly sophisticated and a high degree of automation has been adopted. As a result, X-ray crystallography has become a common tool for biochemists with an interest in structural biology. Single-wavelength anomalous diffraction phasing using $\mathrm{S}$ atoms in native protein molecules, designated S-SAD, is becoming a widely applied technique in protein crystallography. Using this method, it is not necessary to prepare labelled crystals, such as heavy-atom derivatives or selenomethionine-substituted protein crystals. Despite the early suggestion of its availability (Wang, 1985), limited success was reported because of difficulties due to the small anomalous signal of S atoms (Hendrickson \& Teeter, 1981; Dauter et al., 1999; Liu et al., 2000; Bond et al., 2001; Cianci et al., 2001; de Graaff et al., 2001; Gordon et al., 2001; Yang \& Pflugrath, 2001; Brown et al., 2002; Lemke et al., 2002; Li et al., 2002; Micossi et al., 2002; Debreczeni, Bunkóczi, Girmann \& Sheldrick, 2003; Debreczeni, Bunkóczi, Ma et al., 2003; Ramagopal et al., 2003; Lartigue et al., 2004; Sekar et al., 2004; Lakomek et al., 2009).
In the case of S-SAD phasing, use of a longer wavelength is advantageous for detecting the anomalous signal of $\mathrm{S}$ atoms. However, the accuracy of the measured diffraction intensity decreases at longer wavelengths because of the greater absorption and scattering effect. Data collection environments and processing methods specialized for using longer-wavelength X-rays have been discussed (Weiss, Sicker, DjinovicCarugo \& Hilgenfeld, 2001; Weiss, Sicker \& Hilgenfeld, 2001; Yang \& Pflugrath, 2001; Cianci et al., 2008). The standard cryogenic crystal mounting technique (Teng, 1990; Garman \& Schneider, 1997) has been improved with a new method that removes the buffer around the protein crystal before flash freezing of the crystals (Kitago et al., 2005). This capillary-top mounting method, previously referred to as the 'loopless' method, has been used to solve 14 novel structures including unpublished results (Watanabe et al., 2005; Hiyama et al., 2006; Kitamura et al., 2008). To date, however, this method has been carried out manually. The processes of harvesting and flash freezing the crystal are performed using both hands, and the mouth is also used for cryo-solution aspiration. Hence, this manual procedure requires specific skills. Automation of aspiration through a micropipette has been proposed for the routine use of this method (Lavault et al., 2006).

Some automated crystal harvesting devices driven by remote-controlled actuators have been developed (Hiraki et al., 2005; Viola et al., 2007). However, the crystal harvesting 


\section{cryocrystallography papers}

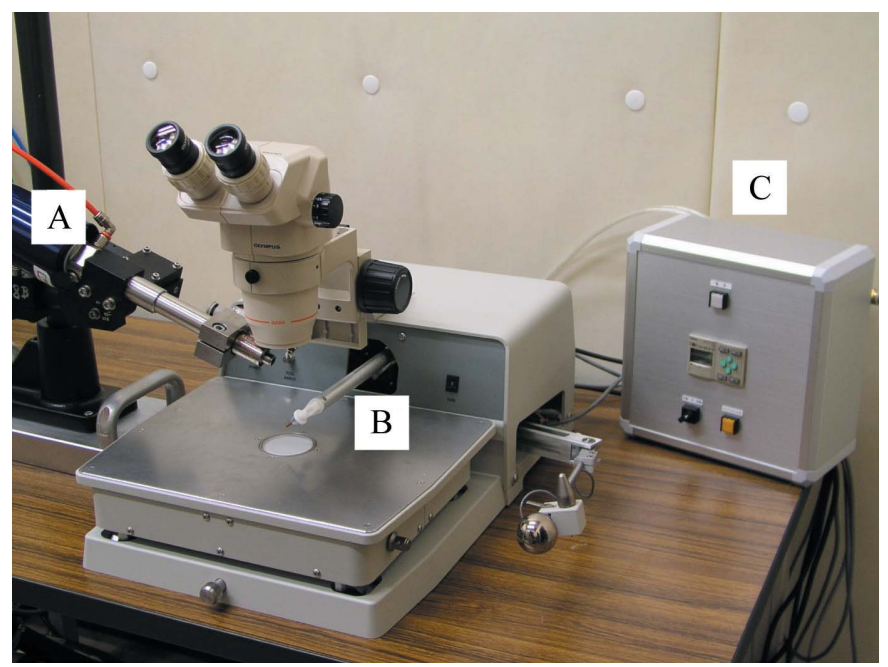

Figure 1

Overall view of the semi-automated crystal mounting device for the capillary-top mounting method. A: cryo-cooler; B: micro-manipulator; C: sequential timer.

operation is quite complicated and it is not easy to automate. Therefore, in our method, the crystal harvesting process is performed manually using a micro-manipulator, and the subsequent procedures have been automated. These developments make it possible to use the capillary-top mounting method routinely for longer-wavelength S-SAD phasing.

\section{Description and operation}

The prototype device (Figs. 1 and 2) was designed as a semiautomated device for crystal handling to allow the protein crystal to be harvested from the crystallization drop with manual movement of a micro-manipulator, and it is subsequently possible to remove the buffer and flash freeze the crystal automatically. The 7200CR Dual Head Epoxy Die Bonder (West-Bond, Anaheim, CA, USA) was chosen as the base component of the device. A magnet to hold the cryo-pin is placed at the head of the mechanically linked X-Y-Z micromanipulator of the bonder, and a cryo-pin with a loop can be put there as the standard goniometer head for cryo protein crystallography (Fig. 2). There is a through-hole at the centre of the magnet where a suction line for removing the solution is connected. This makes it possible to use a custom-made cryopin for the capillary-top mounting method (Watanabe, 2006). In order to facilitate harvesting of the crystal, the tilt angle of the cryo-pin can be adjusted arbitrarily with a motor using an optical encoder attached at the manipulator arm. The docked or resting position of the bonder is used as the crystal freezing position where the conventional cryo-cooler nozzle is placed. The flow of the cryo-stream is gated by an air-driven mechanical shutter similar to those used at many synchrotron facilities (Giraud et al., 2009). It is also possible to use a standard cryo-pin, if it is not necessary to use the capillary-top method.

When the operator has successfully harvested a protein crystal from the crystallization drop, and moves the manipulator up from the drop to the freezing position, a series of

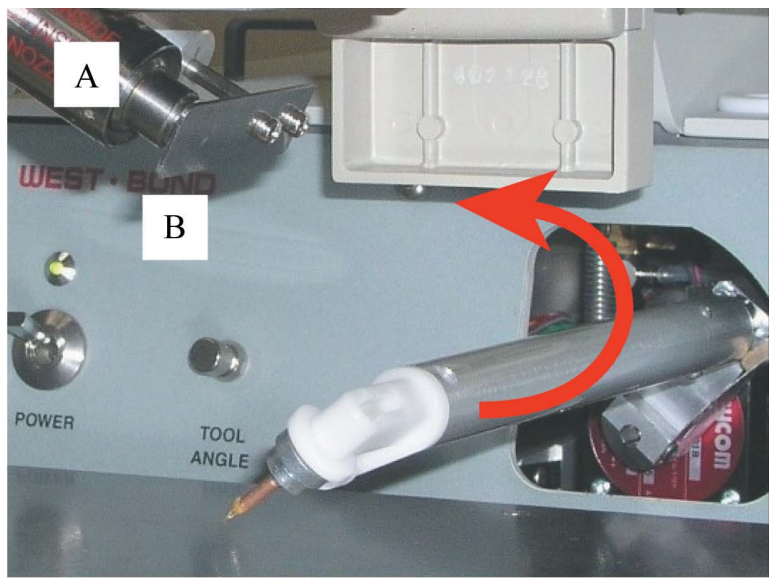

(a)

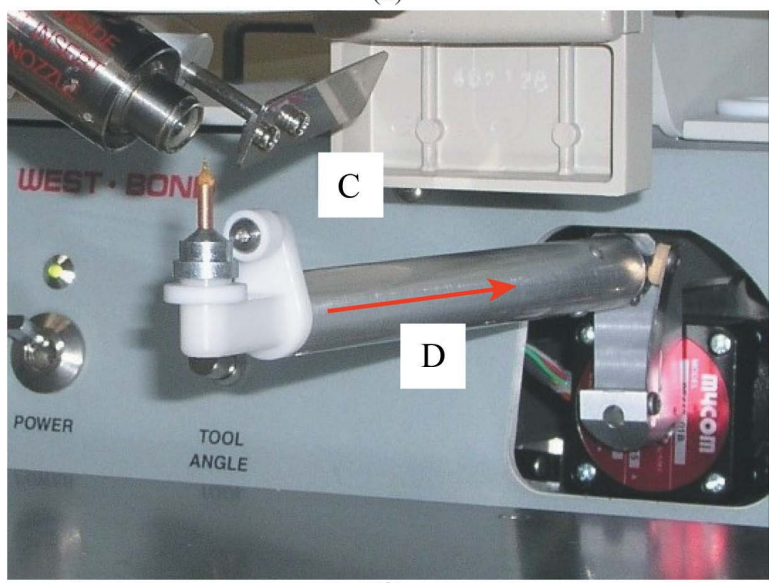

(b)

Figure 2

Magnified view of the micro-manipulator at $(a)$ the crystal harvesting position and $(b)$ the crystal freezing position. A: cryo-cooler nozzle; B: cryo-stream shutter in the closed position; C: cryo-stream shutter in the open position. The suction line is inserted into the manipulator head as indicated by the arrow D. The manipulator head will rotate from the harvesting position to the freezing position as indicated by the anticlockwise arrow when the user has successfully harvested a crystal and releases the control lever of the micro-manipulator.

events controlled by a sequential timer are triggered. A solenoid valve for the suction line opens and the solution around the harvested protein crystal in the loop is immediately aspirated through the line. The shutter then opens and the cryo-stream flash freezes the crystal, the surrounding solution of which has been removed. Typically, the solenoid valve for the suction line opens $100 \mathrm{~ms}$ after the manipulator backs to the freezing position as shown in Fig. 2(b), and the cryo-stream shutter opens a further $100 \mathrm{~ms}$ later. The intervals between sequenced events can be set by the user. Once the crystal is frozen, it is possible to recover the cryo-pin using a conventional cryo-tong, and it can be stored in a liquid nitrogen dewar. Short videos showing these procedures can be downloaded as supplementary data. ${ }^{\mathbf{}}$ This prototype is currently installed at Hokkaido University.

\footnotetext{
${ }^{\mathbf{1}}$ These videos are available from the IUCr electronic archives (Reference: HE5462). Services for accessing these files are described at the back of the journal.
} 


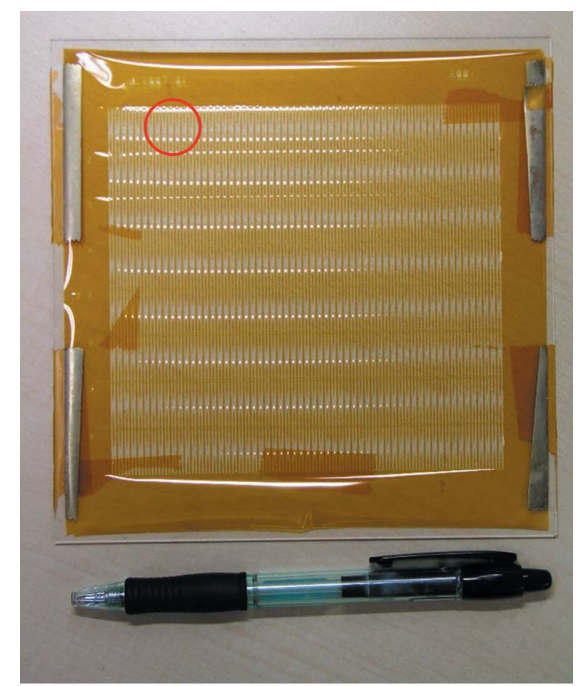

(a)

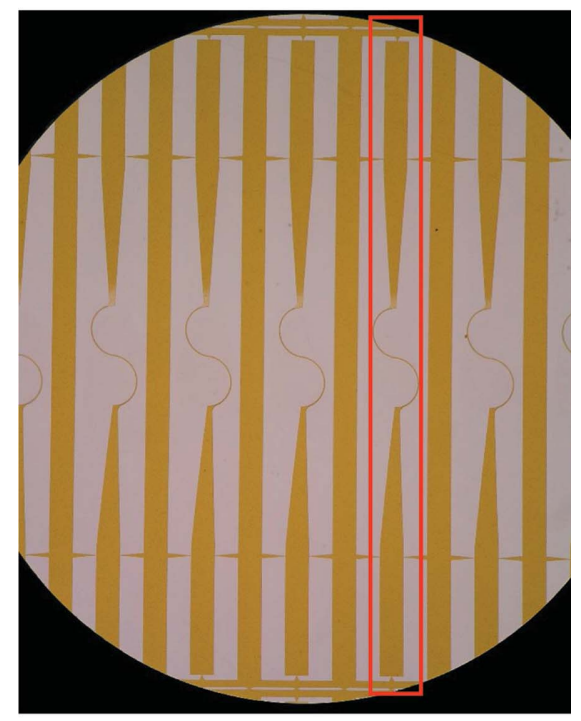

(b)

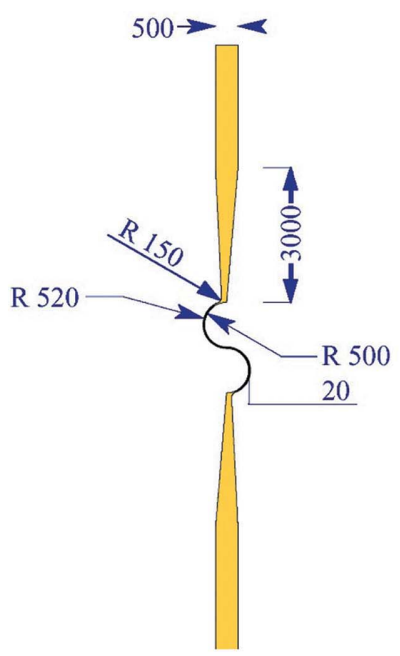

(c)

Figure 3

The microlithography polyimide film with S-shaped design. (a) Photograph of the whole sheet. (b) Magnified view of the sheet indicated by the red circle in $(a)$. The red rectangle corresponds to one loop. (c) Example of dimensions of one S-shaped film for a $1 \mathrm{~mm}$-diameter loop.
The loop of the custom cryo-pin for the capillary-top mounting method was also improved. The conventional nylon loop glued to the tip of the glass capillary (Kitago et al., 2005) was replaced with a microlithography shaped polyimide film (Fig. 3). Polyimide film has already been used as a loop for MicroMounts (Thorne et al., 2003) and LithoLoops (Molecular Dimensions, Suffolk, England, and Protein Wave Corporation, Kyoto, Japan). Using polyimide film has some advantages over the nylon loop, including mechanical stability, negligible X-ray diffraction and absorption, and higher productivity. To locate the loop centre on the capillary axis, which is important to make the protein crystal sit properly on the tip of the capillary as shown in Fig. 4, we use an S-shaped design. To make the loop structure with the $S$-shaped film, one side of the scaffold is attached to the capillary, and then the other side is glued to the opposite side of the capillary, as shown in Fig. 5.

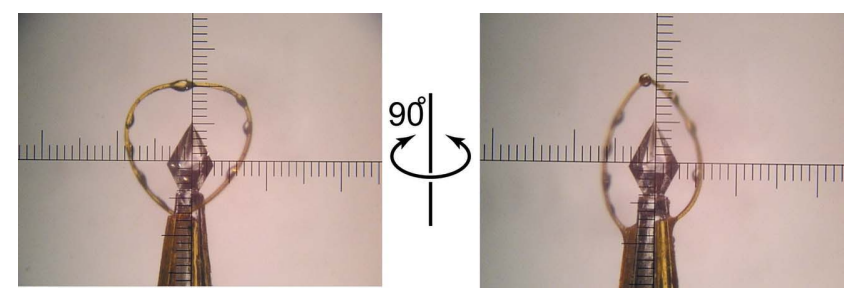

\section{Figure 4}

Examples of a crystal frozen using the newly designed loop. A thaumatin crystal was used. One division of the scale is $50 \mu \mathrm{m}$.

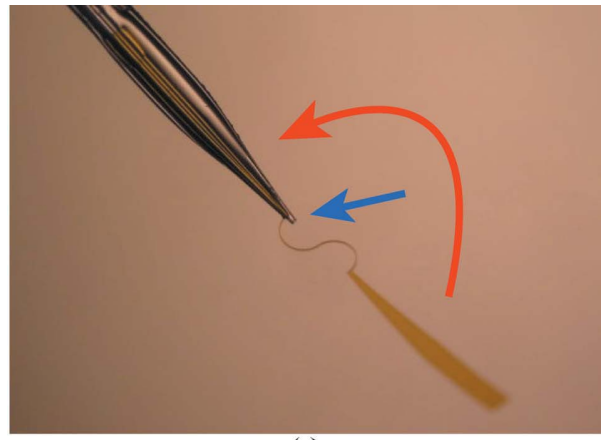

(a)

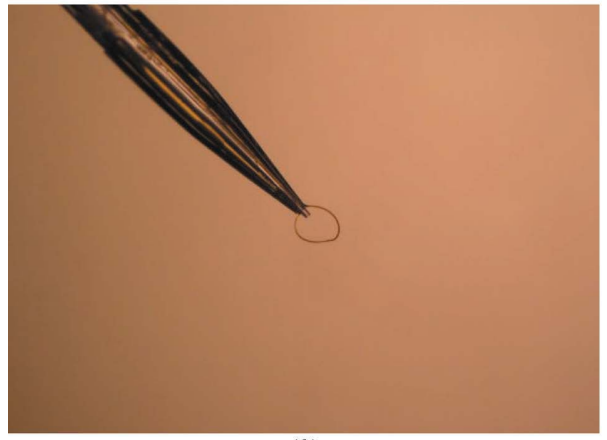

(b)

Figure 5

Procedures for making the loop structure using the S-shaped film. (a) One side of the film scaffold is glued to the capillary, and then the other side is glued to the opposite side of the capillary, as shown in $(b)$. The tip of the capillary protrudes slightly into the $\mathrm{S}$ shape, as indicated by the blue arrow in the photograph. 
Table 1

Diffraction data statistics for a thaumatin crystal.

Values in parentheses are for the highest resolution shell.

\begin{tabular}{ll}
\hline Wavelength $(\AA)$ & 2.2909 \\
Space group & $P 4_{1} 2_{1} 2$ \\
Unit-cell parameters $(\AA)$ & $a=b=57.9, c=150.1$ \\
Resolution limits $(\AA)$ & $50.0-2.17(2.21-2.17)$ \\
Oscillation width $\left(^{\circ}\right)$ & 0.5 \\
No. of unique reflections & 25731 \\
Multiplicity ${ }^{\dagger}$ & $14.2(9.2)$ \\
Completeness $(\%)$ & $99.6(93.0)$ \\
$R_{\text {sym }}$ & $0.070(0.317)$ \\
$I / \sigma(I)$ & $39.8(6.2)$ \\
Wilson $B$ factor & 28.0 \\
\hline
\end{tabular}

$\dagger$ Using 'Scale Anomalous' option of HKL-2000.

\section{Test experiment}

To demonstrate this system, a longer-wavelength X-ray experiment was carried out using a thaumatin crystal as a sample. Thaumatin from Thaumatococcus daniellii (T-7638; Sigma, St Louis, MO, USA) was dissolved in distilled water $\left(30 \mathrm{mg} \mathrm{ml}^{-1}\right)$ without further purification. Crystals were obtained under the conditions described by Ko et al. (1994), using 0.1 MN-(2-acetamido) iminodiacetic acid (ADA) buffer pH 6.5 and $0.6 M$ sodium potassium tartrate. The reservoir solution had a volume of $1 \mathrm{ml}$, and the crystallization drop consisted of $2 \mu \mathrm{l}$ of protein solution and $2 \mu \mathrm{l}$ of reservoir solution. For the diffraction experiment, a crystal of about $0.2 \times 0.2 \times 0.4 \mathrm{~mm}$ was used. The crystal was quick-dipped in cryoprotectant solution containing $30 \%(v / v)$ glycerol before harvesting. It was then mounted by the capillary-top mounting method using the device and the custom cryo-pin. Diffraction data were collected up to a resolution of $2.17 \AA$ using the inhouse chromium X-ray system described previously (Kitago et al., 2005; Watanabe et al., 2005). A total of 720 images with $0.5^{\circ}$ oscillation were collected with a crystal-to-detector distance of $80 \mathrm{~mm}$. The collected intensities were indexed, integrated, corrected for absorption and scaled using HKL-2000 (Otwinowski \& Minor, 1997). The detailed diffraction data statistics are presented in Table 1 . The positions of anomalous scatterers were located using SHELXD (Sheldrick, 2008) at a resolution of $2.4 \AA$ after analysing the substructure structure factors using SHELXC (Sheldrick, 2008). The initial phases were estimated using SHELXE (Sheldrick, 2008) and improved by density modification with a solvent content of 0.52 . The protein model including 4-205 of 211 residues with side chains could be built automatically by $A R P / w A R P$ (Perrakis et al., 1999). Part of an electron density map and an automatically constructed model are shown in Fig. 6.

Recently, Warkentin \& Thorne (2009) have demonstrated that careful removal of external solution around the crystal using hydrophobic oil, such as Paraton-N, makes it possible to freeze the crystal as-grown without cryoprotectant. We have also performed experiments to show the usefulness of our method for mounting crystals without cryoprotectant. Thaumatin powder was dissolved in 0.1 $M$ ADA buffer $\mathrm{pH}$ 6.5. A reservoir solution including $1.5 \mathrm{M}$ sodium potassium tartrate
Table 2

Diffraction data statistics $\left(\AA,^{\circ}\right)$ for crystals frozen using Paraton-N and the capillary-top mounting method without cryoprotectant.

Values in parentheses are for the highest resolution shell.

\begin{tabular}{lllll}
\hline Samples & Paraton 1 & Paraton 2 & Capillary-top 1 Capillary-top 2 \\
\hline Space group & $P 4_{1} 2_{1} 2$ & $P 4_{1} 2_{1} 2$ & $P 4_{1} 2_{1} 2$ & $P 4_{1} 2_{1} 2$ \\
Cell dimensions & $a=b=58.5$, & $a=b=58.4$, & $a=b=58.3$, & $a=b=58.1$, \\
& $c=150.7$ & $c=150.5$ & $c=150.6$ & $c=150.2$ \\
Oscillation range & 10 & 10 & 10 & 10 \\
Oscillation width & 0.5 & 0.5 & 0.5 & 0.5 \\
Resolution limits & $50.0-2.22$ & $50.0-2.22$ & $50.0-2.22$ & $50.0-2.22$ \\
\multicolumn{1}{c}{$(2.26-2.22)$} & $(2.26-2.22)$ & $(2.26-2.22)$ & $(2.26-2.22)$ \\
I/ $(I)$ & $21.0(2.8)$ & $28.1(6.8)$ & $26.0(3.2)$ & $25.1(5.0)$ \\
Mosaicity range & $0.64-0.72$ & $0.68-0.70$ & $0.76-0.77$ & $0.79-0.90$ \\
\hline
\end{tabular}

was used for crystallization following the method of Warkentin \& Thorne (2009). Crystals were frozen by three different procedures: using the standard loop with mother liquor, using the standard loop with Paraton-N and using the capillary-top mounting method. Each crystal was washed with the reservoir solution before freezing, in order to remove precipitate surrounding the crystal. Typical diffraction patterns are shown in Fig. 7. Frozen crystals mounted using our capillary-top mounting method, like those mounted using Paraton-N, do not give ice rings. Four (two for each) data sets with a total oscillation range of $10^{\circ}$ were collected for these two mounting methods using different crystals. The conditions for the diffraction measurements were the same as for the above experiment. As shown in Table 2, the crystals frozen using the capillary-top mounting method are of almost the same quality as those obtained using Paraton-N.

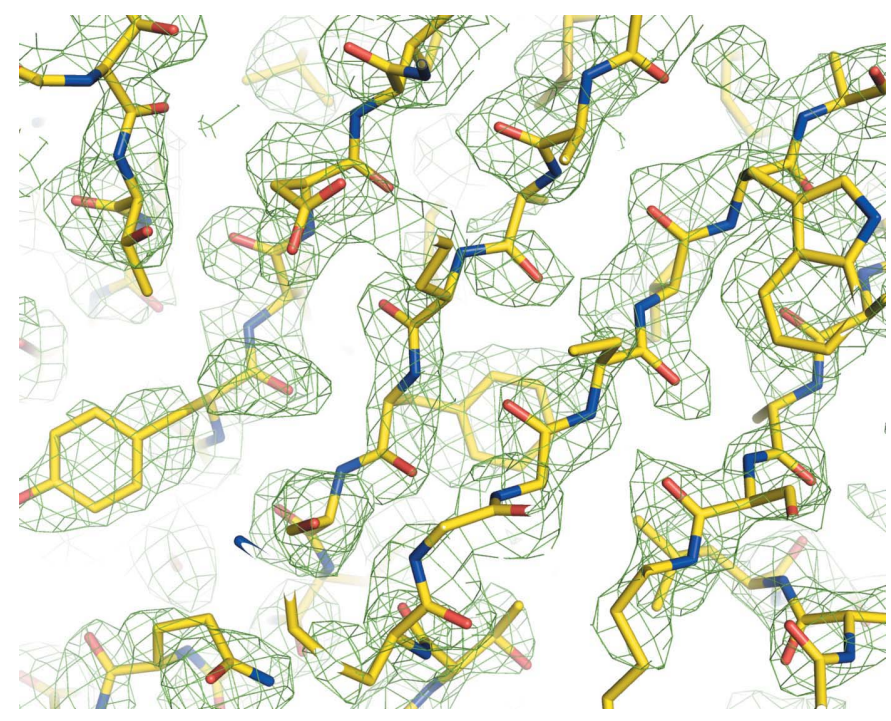

Figure 6

An electron density map and an automatically constructed model of the thaumatin crystal. The green mesh shows the electron density map contoured at the $1.0 \sigma$ level calculated using initial phases determined by longer-wavelength S-SAD with the capillary-top mounting method. The automatically built protein model constructed by the program $A R P /$ $w A R P$ is also shown. 


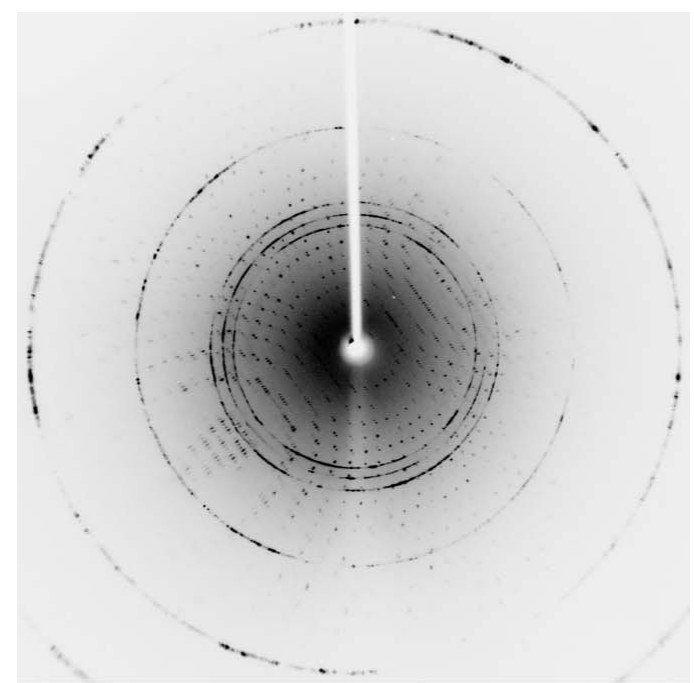

(a)

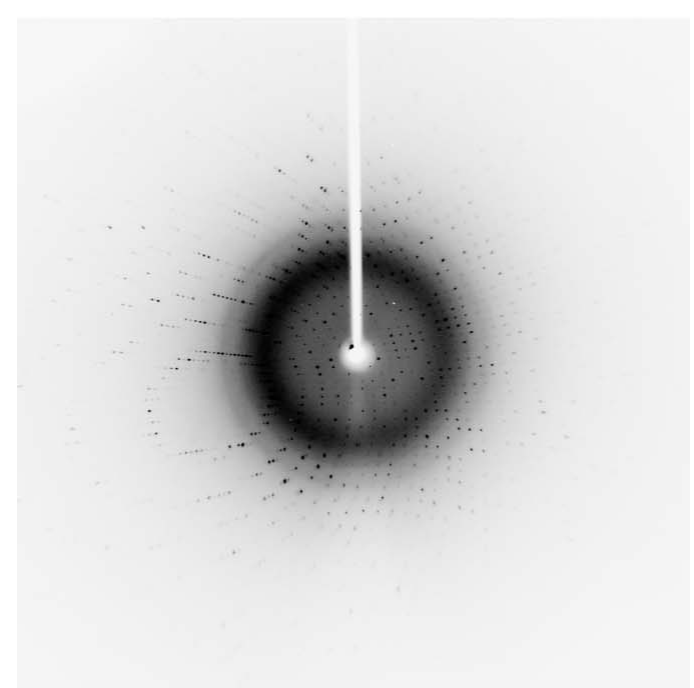

(b)

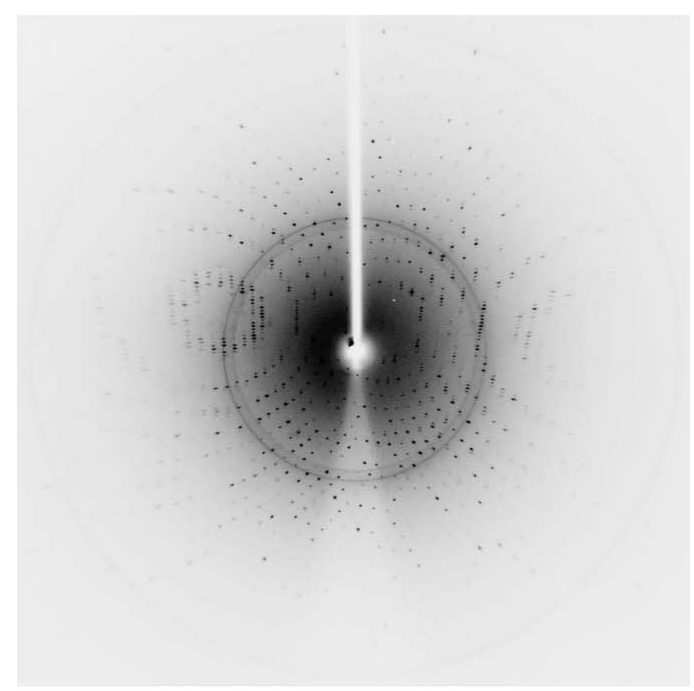

(c)

Figure 7

Examples of diffraction images: (a) crystal frozen using the standard loop with the reservoir solution, $(b)$ crystal frozen using the standard loop with Paraton-N and $(c)$ crystal frozen using the capillary-top mounting method. Images $(b)$ and $(c)$ correspond to 'Paraton 2' and 'Capillary-top 1 ' in Table 2 , respectively.

\section{Discussion and conclusion}

Our capillary-top mounting method is a powerful tool for longer-wavelength S-SAD phasing. The semi-automated device described here has convenient features for crystal mounting that will allow structural biologists to use the S-SAD method with non-labelled protein crystals. The structure of the fluorescent protein KillerRed (Carpentier et al., 2009; Pletnev et al., 2009), the crystal dimensions of which are about $250 \times$ $250 \times 200 \mu \mathrm{m}$, was solved independently by S-SAD using this mounting device (PDB code 3a8s, unpublished results). In addition to the absorption effect, this method can reduce the background noise coming from the frozen cryo-solution. This feature is expected to be applicable to the measurement of micro crystals and ultra-high-resolution X-ray diffraction studies. The bore diameter of the tip of the current handmade capillary is about $40-200 \mu \mathrm{m}$. With these dimensions, there is none of the trouble in removing the external solution that could be caused by the viscosity of the mother liquor. However, the current device is only suitable for crystals larger than $40 \mu \mathrm{m}$. In order to enable use for very small crystals growing in a viscose solution, we are planning to develop a dual capillary device, the conceptual diagram of which is shown in Fig. 8. The idea for the device is functional separation of the capillaries: an inner capillary for crystal holding and an outer capillary for removal of the external solution.

This semi-automated device will also expand application of the capillary-top mounting method. The process of finding a suitable cryoprotectant for freezing a protein crystal is sometimes difficult and time consuming. We have shown that thaumatin crystals prepared using almost the same conditions as used by Warkentin \& Thorne (2009) could be mounted asgrown using our capillary-top mounting method without cryoprotectant. Using hydrophobic oil sometimes causes damage to protein crystals. As our method removes the external solution around the crystal by aspiration, it is not

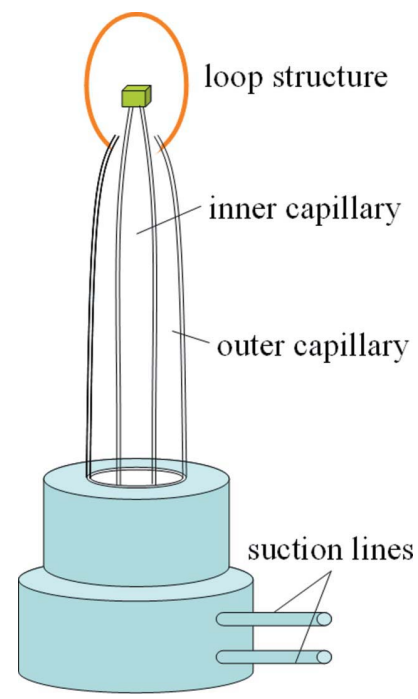

Figure 8

Conceptual diagram of a dual capillary device for mounting a very small crystal grown in a viscose solution. 
necessary to use hydrophobic oil to remove the solution. Qi \& Jiang (2010) have tested 16 lysozyme crystals and 12 were successfully mounted without the use of cryoprotectant. Further experiments will be necessary to generalize the applicability. It was not possible to successfully freeze thaumatin crystals grown from $0.6 M$ sodium potassium tartrate. However, we think that this method is worth attempting for those crystals for which no suitable cryoprotectant can be found.

This capillary-top mounting method also facilitates the automated centring of cryo-cooled crystals. It is sometimes quite difficult to identify the frozen crystal in the lens-shaped frozen liquid. However, a crystal mounted by this method is located easily because the protein crystal will be frozen without surrounding liquid (Lavault et al., 2006). This feature will also be useful to spectroscopic investigations of protein crystals, such as optical monitoring of enzyme kinetics in the crystalline state (De la Mora-Rey \& Wilmot, 2007).

The authors would like to thank Dr A. Sanjo of Protein Wave Corporation for his help in manufacturing the polyimide film. We also thank Mr J. Price of West-Bond Inc. and Mr K. Yoshioka of HiSOL Corporation for modification of the bonder, Mr Y. Maruta of LAB Co. Ltd for the timing box, and Dr N. Matsugaki of the Photon Factory for providing information regarding their crystal annealing shutter system. This work was supported by the Targeted Proteins Research Program (TPRP) of the Ministry of Education, Culture, Sports, Science and Technology, Japan.

\section{References}

Bond, C. S., Shaw, M. P., Alphey, M. S. \& Hunter, W. N. (2001). Acta Cryst. D57, 755-758.

Brown, J., Esnouf, R. M., Jones, M. A., Linnell, J., Harlos, K., Hassan, A. B. \& Jones, E. Y. (2002). EMBO J. 21, 1054-1062.

Carpentier, P., Violot, S., Blanchoin, L. \& Bourgeois, D. (2009). FEBS Lett. 583, 2839-2842.

Cianci, M., Helliwell, J. R. \& Suzuki, A. (2008). Acta Cryst. D64, 1196-1209.

Cianci, M., Rizkallah, P. J., Olczak, A., Raftery, J., Chayen, N. E., Zagalsky, P. F. \& Helliwell, J. R. (2001). Acta Cryst. D57, 1219-1229.

Dauter, Z., Dauter, M., de La Fortelle, E., Bricogne, G. \& Sheldrick, G. M. (1999). J. Mol. Biol. 289, 83-92.

Debreczeni, J. É., Bunkóczi, G., Girmann, B. \& Sheldrick, G. M. (2003). Acta Cryst. D59, 393-395.

Debreczeni, J. E., Bunkóczi, G., Ma, Q., Blaser, H. \& Sheldrick, G. M. (2003). Acta Cryst. D59, 688-696.

De la Mora-Rey, T. \& Wilmot, C. M. (2007). Curr. Opin. Struct. Biol. 17, 580-586.

Garman, E. F. \& Schneider, T. R. (1997). J. Appl. Cryst. 30, 211-237.

Giraud, T., Dobias, F., Gabadinho, J., Rey-Bakaikoa, V., Nurizzo, D., Leonard, G. A. \& Mueller-Dieckmann, C. (2009). J. Appl. Cryst. 42, 125-128.

Gordon, E. J., Leonard, G. A., McSweeney, S. \& Zagalsky, P. F. (2001). Acta Cryst. D57, 1230-1237.
Graaff, R. A. G. de, Hilge, M., van der Plas, J. L. \& Abrahams, J. P. (2001). Acta Cryst. D57, 1857-1862.

Hendrickson, W. A. \& Teeter, M. M. (1981). Nature (London), 290, 107-113.

Hiraki, M., Kato, R., Yamada, Y., Matsugaki, N., Igarashi, N. \& Wakatsuki, S. (2005). Acta Cryst. A61, C149-C150.

Hiyama, T. B., Zhao, M., Kitago, Y., Yao, M., Sekine, S., Terada, T., Kuroishi, C., Liu, Z.-J., Rose, J. P., Kuramitsu, S., Shirouzu, M., Watanabe, N., Yokoyama, S., Tanaka, I. \& Wang, B.-C. (2006). J. Struct. Funct. Genomics, 7, 119-129.

Kitago, Y., Watanabe, N. \& Tanaka, I. (2005). Acta Cryst. D61, 1013 1021.

Kitamura, M., Okuyama, M., Tanzawa, F., Mori, H., Kitago, Y., Watanabe, N., Kimura, A., Tanaka, I. \& Yao, M. (2008). J. Biol. Chem. 283, 36328-36337.

Ko, T.-P., Day, J., Greenwood, A. \& McPherson, A. (1994). Acta Cryst. D50, 813-825.

Lakomek, K., Dickmanns, A., Mueller, U., Kollmann, K., Deuschl, F., Berndt, A., Lübke, T. \& Ficner, R. (2009). Acta Cryst. D65, 220 228.

Lartigue, A., Gruez, A., Briand, L., Blon, F., Bezirard, V., Walsh, M., Pernollet, J. C., Tegoni, M. \& Cambillau, C. (2004). J. Biol. Chem. 279, 4459-4464.

Lavault, B., Ravelli, R. B. G. \& Cipriani, F. (2006). Acta Cryst. D62, 1348-1357.

Lemke, C. T., Smith, G. D. \& Howell, P. L. (2002). Acta Cryst. D58, 2096-2101.

Li, S. et al. (2002). J. Biol. Chem. 277, 48596-48601.

Liu, Z. J., Vysotski, E. S., Chen, C. J., Rose, J. P., Lee, J. \& Wang, B. C. (2000). Protein Sci. 9, 2085-2093.

Micossi, E., Hunter, W. N. \& Leonard, G. A. (2002). Acta Cryst. D58, 21-28.

Otwinowski, Z. \& Minor, W. (1997). Methods Enzymol. 276, 307-326.

Perrakis, A., Morris, R. \& Lamzin, V. S. (1999). Nat. Struct. Biol. 6, 458-463.

Pletnev, S., Gurskaya, N. G., Pletneva, N. V., Lukyanov, K. A., Chudakov, D. M., Martynov, V. I., Popov, V. O., Kovalchuk, M. V., Wlodawer, A., Dauter, Z. \& Pletnev, V. (2009). J. Biol. Chem. 284 32028-32039.

Ramagopal, U. A., Dauter, M. \& Dauter, Z. (2003). Acta Cryst. D59, 1020-1027.

Qi, J.-X. \& Jiang, F. (2010). Chin. Phys. B, 19, 010601.

Sekar, K., Rajakannan, V., Velmurugan, D., Yamane, T., Thirumurugan, R., Dauter, M. \& Dauter, Z. (2004). Acta Cryst. D60, 15861590.

Sheldrick, G. M. (2008). Acta Cryst. A64, 112-122.

Teng, T.-Y. (1990). J. Appl. Cryst. 23, 387-391.

Thorne, R. E., Stum, Z., Kmetko, J., O’Neill, K. \& Gillilan, R. (2003). J. Appl. Cryst. 36, 1455-1460.

Viola, R., Carman, P., Walsh, J., Miller, E., Benning, M., Frankel, D., McPherson, A., Cudney, B. \& Rupp, B. (2007). J. Appl. Cryst. 40, 539-545

Wang, B. C. (1985). Methods Enzymol. 115, 90-112.

Warkentin, M. \& Thorne, R. E. (2009). J. Appl. Cryst. 42, 944-952.

Watanabe, N. (2006). Acta Cryst. D62, 891-896.

Watanabe, N., Kitago, Y., Tanaka, I., Wang, J., Gu, Y., Zheng, C. \& Fan, H. (2005). Acta Cryst. D61, 1533-1540.

Weiss, M. S., Sicker, T., Djinovic-Carugo, K. \& Hilgenfeld, R. (2001). Acta Cryst. D57, 689-695.

Weiss, M. S., Sicker, T. \& Hilgenfeld, R. (2001). Structure, 9, 771777

Yang, C. \& Pflugrath, J. W. (2001). Acta Cryst. D57, 1480-1490. 